
\title{
$\angle$ Research Square \\ Finite Element Analysis of Different Percutaneous Fixation Strategies Used for the Treatment of Tile C1.2 Pelvic Fracture
}

changqing $\mathrm{Xu}$

Doctor

wanbo Zhu

The First Affiliated Hospital of China University of science and technology

lei Liu

The First Affiliated Hospital of China University of science and technology

jiazhao Yang

The First Affiliated Hospital of China University of science and technology

shiyuan Fang ( $\sim$ fangshiyuan2008@126.com )

Anhui Provincial Hospital, Wannan Medical College https://orcid.org/0000-0003-3799-6772

Research article

Keywords: Pelvic fractures, Infix technology, Sacroiliac screw, Biomechanics, Finite element analysis

Posted Date: July 21st, 2020

DOl: https://doi.org/10.21203/rs.3.rs-41960/v1

License: (9) This work is licensed under a Creative Commons Attribution 4.0 International License. Read Full License 


\section{Abstract \\ Objectives}

We evaluated the biomechanical outcomes of 9 different percutaneous fixation strategies used for the fixation of Tile $\mathrm{C} 1.2$ pelvic fracture. This may help surgeons choose the optimal therapy for the unstable pelvic fracture.

\section{Methods}

Tile C1.2 pelvic fractures and implant models were constructed using finite element analysis (FEA) software. Models were assembled for different fracture fixation types. After meshing the models' elements, we used Abaqus software to perform the FEA. Values of the von Mises Stress and model displacement were used to capture the mechanical factors.

\section{Results}

The FEA shows that under axial loading and external rotational loading of $500 \mathrm{~N}$, the combination of an S1 screw and the Infix system provide strong stability, and that an increased number of screws to fix the anterior pelvic ring increased stability.

\section{Conclusion}

This study indicates that a 3-screw-Infix system on the healthy side, plus an S1 sacroiliac screw improves stability of the Tile C1.2 fracture.

\section{Introduction}

Crush injuries and car accident injuries are often accompanied by pelvic fractures. Pelvic fractures are complicated traumas that are mostly caused by high-energy injuries. Half or more associated with different co-morbidities or multiple injuries, the most serious of which are traumatic hemorrhagic shock and pelvic injuries. Each case is unique, providing a challenge for the surgeon to provide the optimal care and fixation technique. Anterior pelvic ring fractures are the most common pelvic fractures. Simple pubic branch fractures can often be treated conservatively. In some patients with unstable pelvic fractures with posterior ring injury, the anterior ring also needs to be treated while the posterior ring is fixed [1]. There are many ways to treat the anterior ring, such as the Stoppa approach, the ilioinguinal approach, and Infix technology. However, for some patients with multiple injuries, patients who are unable to tolerate open reduction and internal fixation with a plate, or those who are in an acute stage and unwilling to accept plate fixation due to social factors, Infix technology is often the first choice, and has been shown to achieve good clinical results. Surgeons have improved their technique in using traditional Infix technology 
in recent years. For example, in the traditional two-nail Infix technology, an additional nail is added to the joint nodules of the pubic bone (the healthy side and the affected side), and sometimes four nails are added on both sides. The best clinical outcomes when stabilizing anterior ring injuries in pelvic fractures have not been systematically studied. For this reason, the author created three-dimensional finite element models to simulate the Tile C1.2 pelvic fracture, and calculate the biomechanical effect of different combinations of Infix technology on the treatment of Tile $\mathrm{C} 1.2$ pelvic fractures. These models can provide some reference for clinical practice.

\section{Materials And Methods}

\subsection{Research Object}

One volunteer (48 years, male) was selected for X-ray and B-ultrasound to rule out pelvic abnormalities, injuries, osteoporosis, tumors, and other relevant factors. A routine 64-slice spiral CT scan was performed with a slice thickness of $1 \mathrm{~mm}$. Data were collected in DICOM format.

\subsection{Research Methods}

We simulated Tile C1.2 pelvic fractures, which are characterized by a sacroiliac joint dislocation and pubic fracture.

\subsubsection{Image processing phase}

The DICOM data were imported into MIMICS 16.0 software. We defined the coronal plane, sagittal plane and transverse plane in the software, and reconstructed a three-dimensional model of the pelvis using the protocols of region growth (Figure 1). We stored the binary STL file to use in subsequent processing.

\subsubsection{Establishment of an unstable pelvic fracture model}

A model of the left pubic fracture was created in three dimensions. The dislocation of the sacroiliac joint in the posterior ring was simulated by removing the ligaments.

\subsubsection{Mesh modification and ligament reconstruction}

The STL file was imported into the 3-MATIC software to modify the mesh and model the anterior and posterior sacroiliac ligaments (Figure 2), the sacrotuberous ligament and the sacrospinous ligament. The final file was exported in STL format.

\subsubsection{Model fixation and experimental classification}

Implant models were established in UG 8.5 software. The assembly process for internal fixation in the fracture models also was performed with UG software. We established 9 fixation models according to the fixation types (Table 1םFigure 3). 
Table 1: model grouping

\begin{tabular}{|l|l|}
\hline Model 1 & blank" group that was not fixed \\
\hline Model 2 & Infix fixation model \\
\hline Model 3 & 3-screw-Infix model with one screw fixed on the affected pubic ramus \\
\hline Model 4 & 3-screw-Infix model with one screw fixed on the healthy pubic ramus \\
\hline Model 5 & 4-screw-Infix model with two screws fixed bilaterally on the pubic ramus \\
\hline Model 6 & S1 sacroiliac screw fixation model \\
\hline Model 7 & combined fixation types from Models 2 and 6 \\
\hline Model 8 & combined fixation types from Models 3 and 6 \\
\hline Model 9 & combined fixation types from Models 4 and 6 \\
\hline Model 10 & combined fixation types from Models 5 and 6 \\
\hline
\end{tabular}

\subsubsection{Mesh transformation (Table 2)}

All the models were meshed using HyperMesh 11.0 software (Altair Engineering, Inc, USA), which was also used to convert from surface mesh to volume mesh.

Table 2: Elements and nodes of the models in this study

\begin{tabular}{lllllll}
\hline \multicolumn{3}{c}{ Element } & \multicolumn{3}{c}{ Nodes } \\
\hline Model1 & $\begin{array}{l}\text { Pelvis } \\
1769595\end{array}$ & Infix & S1 screw & $\begin{array}{l}\text { Pelvis } \\
\text { Snfix }\end{array}$ & S1 screw \\
Model2 & 1767251 & 81972 & & 347409 & & \\
\hline Model3 & 1771089 & 82477 & & 348041 & 128674 & \\
Model4 & 1768532 & 82489 & & 347213 & 128774 & \\
\hline Model5 & 1769577 & 83530 & & 347358 & 129599 & \\
\hline Model6 & & & 1752798 & & & 343984 \\
\hline Model7 & 1762146 & 81972 & 30332 & 347213 & 127411 & 46317 \\
\hline Model8 & 1770021 & 82477 & 30332 & 347212 & 128674 & 46317 \\
\hline Model9 & 1762172 & 82489 & 30332 & 346725 & 128774 & 46317 \\
\hline Model10 & 1755099 & 83530 & 30332 & 344871 & 129599 & 46317 \\
\hline
\end{tabular}

1.2.6 Material properties and boundary conditions (Table 3)

Frictional contact interactions were assumed between the different parts of the models. The interface of the S1 screw and the sacral bone structure was set as equivalent. The interface of the S1 screw polished rod and sacral bone structure was simulated using contact pairs with a friction factor of 0.3. The Infix screw and the stem were set to be equivalent, as was the Infix screw and bone structure. The interface between the fragments was simulated by contact pairs with a friction factor of 0.3. 
Table3: Material properties used in the current study.

\begin{tabular}{ccc}
\hline Material name & Young's modulus $₫ \mathrm{MPa} \square$ & Poisson's ratio \\
\hline Anterior sacroiliac ligament & 208 & 0.2 \\
Interosseous ligaments & 2300 & 0.2 \\
Posterior sacroiliac ligament & 3000 & 0.2 \\
Short posterior sacroiliac ligament & 3000 & 0.2 \\
Sacrotuberous ligament & 46 & 0.2 \\
Sacrospinous ligament & 46 & 0.2 \\
Infix-screw & 110000 & 0.3 \\
\hline
\end{tabular}

The bone tissue was modeled as an inhomogeneous, isotropic and elastic-plastic material. The material properties were determined by the apparent density of bone tissue $\rho\left(\mathrm{g} / \mathrm{cm}^{3}\right)$. This density was determined by the gray level from the CT scans. A linear relationship between Hounsfield units and grayscale was assumed, and we chose the following density-elasticity equation to convert apparent density into Young's modulus (E, in MPa). The Poisson's ratio of the femur was assigned as 0.3.

All the FE models were subjected to a load of $500 \mathrm{~N}$ applied superior to S1 to assume full weightbearing in patients. External rotation was simulated by applying $500 \mathrm{~N}$ to the left anterior superior iliac spine.

\section{Results}

The Axial Load Analysis

2.1 von Mises stress distribution.

The maximum stress distribution of the 10 models with $500 \mathrm{~N}$ axial loading is shown in Table 4 . With different combinations of Infix fixation, the stress on the whole pelvic ring changed slightly. The Iinfix combination with 4 nails changed the most (124.145 MPa). When the posterior pelvic ring is fixed with the iliac screw, the overall pelvic ring stress is significantly reduced, but there is no significant difference with the combination of different forms of Infix fixation. Infix fixation without the left sacroiliac screw can also play a role in which the minimum stress can be reduced to $15.498 \mathrm{MPa}$ (Model 4). Model 6 with a simple sacroiliac screw shows better results (stress reduction to $11.621 \mathrm{MPa}$ ). The improvement is more significant when combined with Infix technology (reduction to 9.428MPa). The stresses from the fractures also are reduced when the pelvis is fixed with Infix plus a pubic ramus screw: Model 1 (7.959 MPa) > Model $2(5.316 \mathrm{MPa})>\operatorname{Model} 3(3.685 \mathrm{MPa})>\operatorname{Model} 4(2.545 \mathrm{MPa})>\operatorname{Model} 5(2.374 \mathrm{MPa})$. A sacroiliac screw also disperses some stress leading to lower stresses: Model 1 (7.959 MPa) > Model 6 
(5.132 MPa). But the stress reduction is larger when using the 4-screw-Infix system and an S1 sacroiliac screw (Model 10), leading to a stress of only 1.743 MPa on the fracture segments. The results indicate that the sacroiliac screw disperses some stresses that are transferred among fragments. Simple fixation of the anterior pelvic ring does not stabilize pelvic fracture. When the posterior pelvic ring is fixed by the $\mathrm{S} 1$ sacroiliac screw, the facture model is stable. However, maximum stress on the fracture segments indicates that Model 8 (2.711 MPa) > Model 9 (1.892 MPa) > Model 10 (1.743 MPa). Stresses between the 3-screw-Infix model and the 4-screw-Infix model are not very large, but differences between Model 9 and Model 8 are greater.

Table 4: The peak von Mises stress $\square$ MPa $l$ of different parts of all models under axial loading.

\begin{tabular}{cccc}
\hline & Pelvic model & Left sacroiliac joint & Fracture sit \\
\hline Model 1 & 157.326 & 28.574 & 7.959 \\
Model 2 & 144.516 & 15.613 & 5.316 \\
Model 3 & 140.433 & 15.547 & 3.685 \\
Model 4 & 139.724 & 15.532 & 2.545 \\
Model 5 & 124.145 & 15.498 & 2.374 \\
Model 6 & 66.069 & 11.621 & 5.132 \\
Model 7 & 58.607 & 9.632 & 3.146 \\
Model 8 & 57.765 & 9.594 & 2.711 \\
Model 9 & 58.143 & 9.587 & 1.892 \\
Model 10 & 51.059 & 9.428 & 1.743 \\
\hline
\end{tabular}

\subsection{Displacement analysis}

The displacement of the nine different models under a $500 \mathrm{~N}$ vertical load is shown in Table 5. We found that total displacement, vertical displacement and horizontal displacement are reduced on the pelvis, left sacroiliac joint and fracture site displacement compared to Model 1. When the posterior pelvic ring is fixed with the sacroiliac screw, displacement is reduced by a large amount. With the combination of Infix and a sacroiliac screw, displacement reduction is not very large. Of all the Infix alone models, parameters of total displacement, vertical displacement and horizontal displacement are in the order of Model $5<$ Model $4<$ Model $3<$ Model 2. With addition of the sacroiliac screw, displacement parameters are in the order of Model $10<$ Model $9<$ Model $8<$ Model 7. The fixation model with 4-screw-Infix in addition to a sacroiliac screw shows the least displacement. The detailed values for displacement in each model are shown in Table 5.

Table 5: The peak displacement (mm\of different part of all models under axial loading. 


\begin{tabular}{llllllllll}
\hline & Pelvic & \multicolumn{4}{c}{ Left sacroiliac joint } & \multicolumn{3}{l}{ Fracture site } \\
\hline Model 1 & total & vertical & horizontal & total & vertical & horizontal & total & vertical & horizontal \\
Model 2 & 3.428 & 3.479 & 0.287 & 3.78 & 2.943 & 0.188 & 1.726 & 1.704 & 0.074 \\
Model 3 & 3.351 & 2.762 & 0.163 & 1.794 & 1.361 & 0.081 & 0.661 & 0.526 & 0.027 \\
Model 4 & 3.296 & 2.785 & 0.158 & 1.769 & 1.324 & 0.074 & 0.347 & 0.301 & 0.026 \\
Model 5 & 3.209 & 2.523 & 0.146 & 1.513 & 1.187 & 0.065 & 0.315 & 0.173 & 0.022 \\
Model 6 & 2.847 & 1.856 & 0.153 & 1.634 & 1.258 & 0.07 & 1.022 & 0.985 & 0.039 \\
Model 7 & 2.612 & 1.643 & 0.118 & 1.258 & 0.912 & 0.053 & 0.562 & 0.538 & 0.013 \\
Model 8 & 2.556 & 1.687 & 0.102 & 1.196 & 0.869 & 0.049 & 0.318 & 0.319 & 0.013 \\
Model 9 & 2.625 & 1.464 & 0.089 & 1.147 & 0.821 & 0.046 & 0.225 & 0.204 & 0.012 \\
Model 10 & 2.493 & 0.997 & 0.075 & 0.925 & 0.676 & 0.037 & 0.197 & 0.197 & 0.01 \\
\hline
\end{tabular}

External Rotational Load Analysis

\subsection{Overall stress analysis}

The maximum stress distribution of the 10 models at $500 \mathrm{~N}$ loading on the left anterior superior iliac spine is shown in Table 6. When the anterior pelvic ring is fixed with Infix, the overall pelvic ring stress of Models 2-5 is in the range of 149.602-158.914 MPa, which is similar to Model 1 (162.581 MPa). Model 6 with S1 sacroiliac screw fixation alone has a lower stress of $120.521 \mathrm{MPa}$. With the combination of the S1 screw and Infix, the overall pelvic ring stress of Models 7-10 is in the range of 99.471-113.354 MPa. Model 10 shows the least stress. Similarly, the stress on the left sacroiliac joint is more than $30 \mathrm{MPa}$ when the anterior ring is fixed. While the posterior ring is fixed by the S1 screw, stress on the left sacroiliac is reduced to $26.431 \mathrm{MPa}$. The combination between Infix and the S1 screw reduces the stress further. The results indicate that when the pelvis is subjected to external rotation stress, none of the Infix fixations effectively fix the unstable Tile C1.2 fracture. But the fixation of the posterior ring may provide enough stability. The use of simple sacroiliac screws improves the overall pelvic ring and sacroiliac joints. The effect is better when fixed with Infix. There are differences depending on the number of screws on the pubic branch of Infix. We find that 4 screws provide the best results.

Table 6: The peak von Mises stress $\square \mathrm{MPa}$ of different parts of all models under external rotational loading. 


\begin{tabular}{ccc}
\hline & Pelvic model & Left sacroiliac joint \\
\hline Model 1 & 162.581 & 42.728 \\
Model 2 & 158.914 & 34.256 \\
\hline Model 3 & 152.736 & 33.632 \\
\hline Model 4 & 155.210 & 33.135 \\
\hline Model 5 & 149.602 & 32.947 \\
\hline Model 6 & 120.521 & 26.431 \\
\hline Model 7 & 113.354 & 22.434 \\
\hline Model 8 & 109.579 & 21.576 \\
\hline Model 9 & 104.587 & 21.093 \\
\hline Model 10 & 99.471 & 20.454 \\
\hline
\end{tabular}

\subsection{Displacement analysis}

The values of displacement of the pelvis, the left sacroiliac joint, and the pubic branch fracture of the 10 Models under external rotational loading are shown in Table7. When we compare Infix among fixation types represented by Models 2-5, the overall pelvic displacement and sacroiliac joint fixation with the 4 screw Infix was the lowest. When the pelvis was fixed only with an S1 screw in Model 6, displacement of the overall pelvis, sacroiliac joint and pubic branch fracture site are $3.473 \mathrm{~mm}, 0.258 \mathrm{~mm}$ and $1.007 \mathrm{~mm}$, respectively. Model 10, with an S1 screw and the 4-screw-Infix type, had displacements of the overall pelvis, sacroiliac joint and pubic branch fracture site of $2.412 \mathrm{~mm}, 0.149 \mathrm{~mm}$ and $0.017 \mathrm{~mm}$, respectively. The results indicate that under external rotational loading, Tile C1.2 fractures can be fixed effectively by simply using sacroiliac screw fixation.

Table 7: The peak displacement (mm】of different parts of all models under external rotational loading.

\begin{tabular}{cccc}
\hline & Pelvic model & Left sacroiliac joint & Fracture site \\
\hline Model 1 & 6.451 & 0.898 & 2.285 \\
Model 2 & 5.335 & 0.447 & 1.561 \\
\hline Model 3 & 4.575 & 0.235 & 1.024 \\
\hline Model 4 & 4.49 & 0.272 & 0.086 \\
\hline Model 5 & 3.146 & 0.243 & 0.073 \\
\hline Model 6 & 3.473 & 0.258 & 1.007 \\
\hline Model 7 & 2.987 & 0.196 & 0.066 \\
\hline Model 8 & 2.751 & 0.163 & 0.034 \\
\hline Model 9 & 2.694 & $0.155 \square$ & 0.019 \\
\hline Model 10 & 2.412 & 0.149 & 0.017 \\
\hline
\end{tabular}

\section{Discussion}

It is well known that unstable pelvic ring fractures require surgical treatment, but the selection of the ideal surgical scheme has been the subject of many controversies [2-4]. Surgeons often focus on the restoration of the stability of the posterior pelvic ring and neglect the repair of structural damage to the 
anterior pelvic ring [5]. Hill et al. [6] showed that although the death rate 1 year after pubic branch fracture injury is lower than that of hip fracture, but the mortality rate after 5 years tends to be the same. This may be related to long-term passive braking and persistent pain. This undesirable situation usually occurs in patients with posterior ring damage that is not detected initially. At present, a general view is that any types of anterior and posterior ring injuries should be fixed in order to enable early movement and prevent postoperative displacement $[1,7]$. Common pelvic fracture fixation is divided into internal fixation and external fixation, but current research shows that compared with more invasive internal fixation, minimally invasive reduction may have some benefits for patients $[8,9]$. The amount of bleeding, soft tissue injury and the incidence of nerve injury have improved. On the other hand, various internal fixation methods are more challenging for surgeons. In clinical practice, most of the minimally invasive techniques choose Infix technology for fixation. A variety of combinations of Infix technology have been produced, but there are not many biomechanical studies on unstable pelvic fractures. Due to the source of the cadaver specimen and its inherent variability, we performed a three-dimensional finite element analysis and $d$ the stability of different percutaneous fixation methods for the treatment of Tile C1.2 fractures. Finite element models have many potential limitations, but they do provide the advantages of repeatable and comparable biomechanical tests [10].

The results indicate that Infix fixation strategy, regardless of the combination or distribution of screws on the pubic ramus, does not play a decisive role in stability of the unstable posterior ring. The S1 sacroiliac screw can fix effectively the posterior pelvic ring in cases of pelvic fracture, and on some level it improves the anterior pelvic ring. The combination of the S1 screw and the Infix system provides strong stability with additional screws to fix the anterior pelvic ring, increasing stability. Therefore, this present study shows that 3-screw-Infix plus the S1 screw exhibit the best effect. Pelvic fractures like Tile $\mathrm{C} 1.2$ are characterized by a unilateral posterior arch through the sacroiliac joint with complete disruption of the posterior arch, and an anterior ring fracture that needs to be fixed by the Infix system. The iliac screw combined with Infix is an effective and minimally invasive alternative to the treatment of Tile $\mathrm{C} 1.2$ fractures. According to the principle of trauma minimization, it is recommended to use the iliac screw +3 nail Infix fixation on the healthy side. However, this needs further in-vivo study to verify that it will result in positive clinical outcomes.

\section{Declarations}

\section{Data Availability}

The datasets used and/or analyzed during the current study are available from the corresponding author on reasonable request.

\section{Consent}

This study obtained consent to publish from the participants to report individual patient data (CT image data). 
Acknowledgements

We would like to thank Prof. Shiyuan Fang who provided encouragement to write this paper and gave valuable advice.

\section{Authors' contributions}

CX wrote the manuscript. WZ and LL revised and edited the manuscript. CX $\square$ JY and SF participated in the design of the study. All authors have read and approved the manuscript.

\section{Funding}

This study was supported by the Project of Natural Science Foundation of Anhui Province (Grant Nos. 1908085QH359). Authors were funded in the design of the study, collection and analysis of data, the writing of manuscript, and polishing of English manuscripts with mother tongue.

\section{Conflicts of Interest}

The authors declare that they have no conflicts of interest Journal of orthopaedic surgery and research.

\section{References}

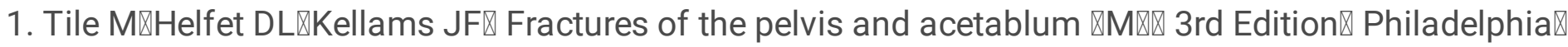
Lippincott Williams $₫$ Wilkins $₫ 2003 \otimes 131 \rrbracket 167 \rrbracket$

2. Gardner MJ. Stabilization of unstable pelvic fractures with supraacetabular compression external fixation. J Orthop Trauma. 2007;21(4):269-73.

3. Jain M, Nanda SN, Mohapatra SS, Samal BP. Bladder incarceration following anterior pelvic infix of a traumatic pubic symphysis diastasis treated with immediate open reduction and internal fixation. $J$ Clin Orthop Trauma. 2017;8(Suppl 1):S11-6. https ://doi.org/10.1016/j.jcot.2017.06.010 (Epub 2017/09/08. PubMed PMID: 28878532; PubMed Central PMCID: PMCPMC5574869).

4. Tile M. Pelvic ring fractures: should they be fixed? J Bone Joint Surg Br. 1988;70(1):1-12.

5. Liu Min, Zhou Xiaosai, Wang Juncheng, Liu liangle, Wang Weiliang, Cai Chunyuan, Yang Guojing. Finite element analysis of anterior ring injury in the treatment of unstable pelvic fracture by different methods [J]. China bone injury, 2019,32 (02): 156-160

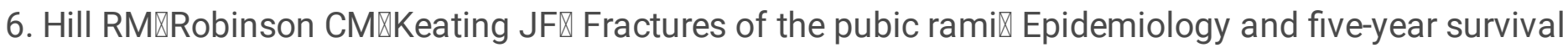

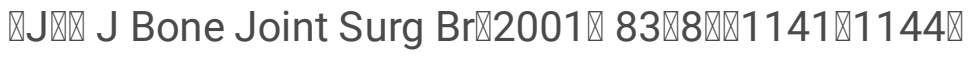

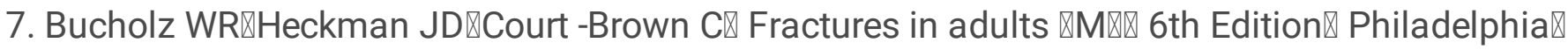
Lippincott Williams $₫$ Wilkins $₫ 2006 \rrbracket 1665 \rrbracket 1714 \rrbracket$

8. Shetty AP, et al. Midterm radiologic and functional outcomes of minimally-invasive fixation of unstable pelvic fractures using anterior internal fixator (INFIX) and percutaneous iliosacral screws. $J$ 
Clin Orthop Trauma. 2017;8(3):241- 8. https ://doi.org/10.1016/jjcot 201705009 (Epub 2017 Jun 10).

9. Hiesterman TG, et al. Surgical technique: a percutaneous method of subcutaneous fixation for the anterior pelvic ring: the pelvic bridge. Clin Orthop Relat Res. 2012;470(8):2116-23. https ://doi.org/10.1007/s1199 9-012-2341-4.

10. Becker, C.A., Kammerlander, C., Kußmaul, A.C. et al. Modified less invasive anterior subcutaneous fixator for unstable Tile-C-pelvic ring fractures: a biomechanical study. BioMed Eng OnLine 18, 38 (2019) doi:10.1186/s12938-019-0648-z

\section{Figures}

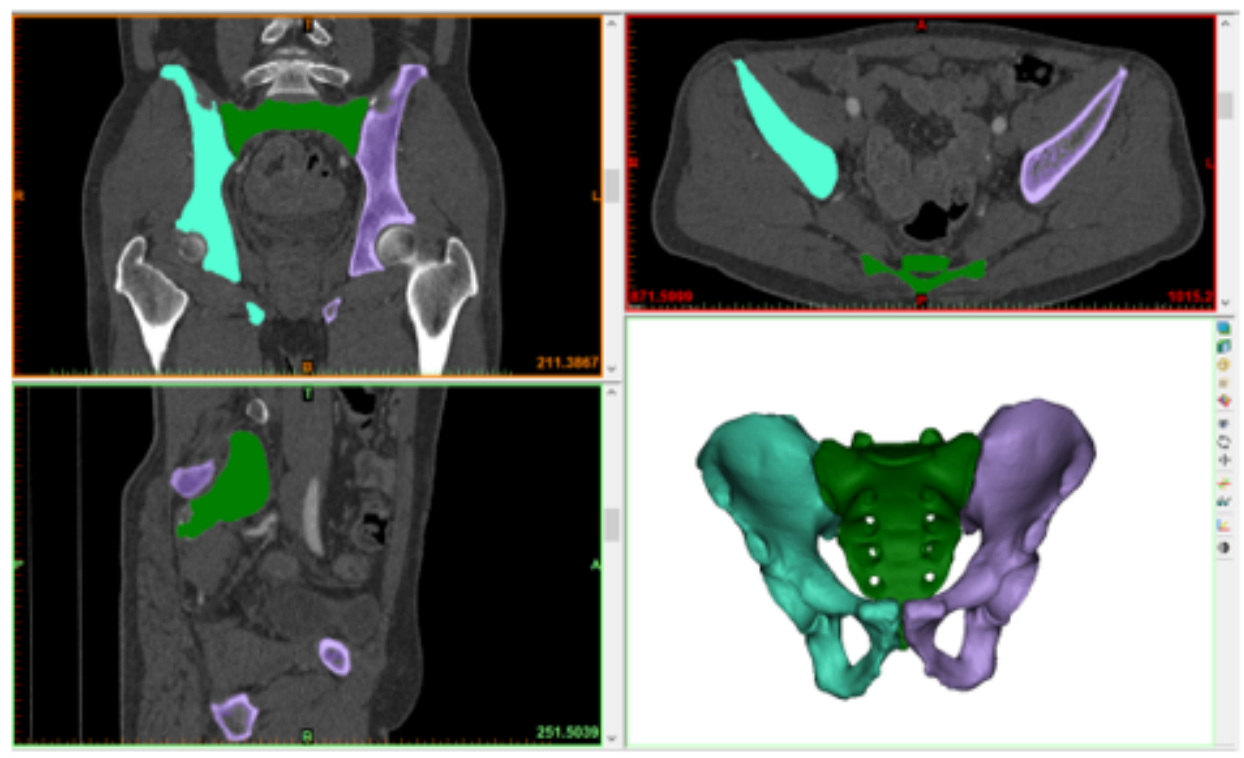

\section{Figure 1}

$\mathrm{CT}$ (computed tomography) data image
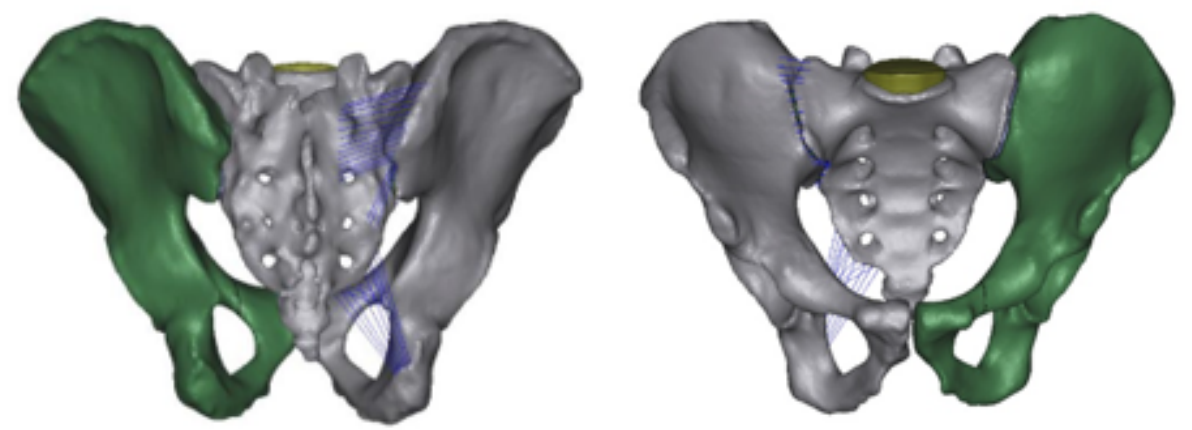

Figure 2

Mesh modification and adding ligaments 


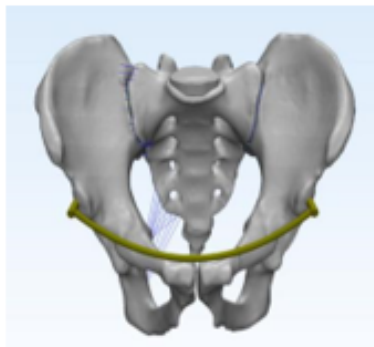

Model1

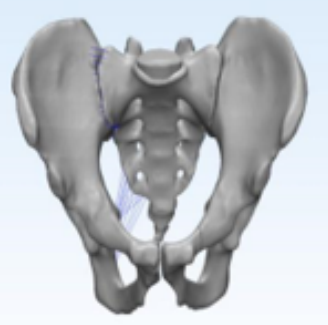

Mode12

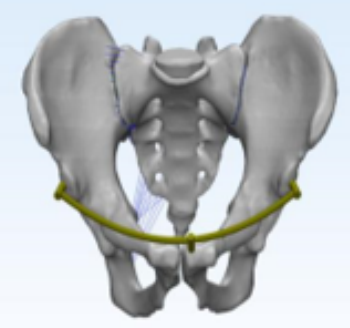

Mode13

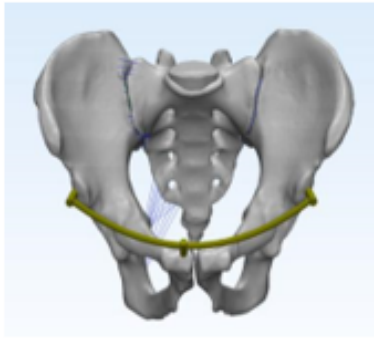

Model4

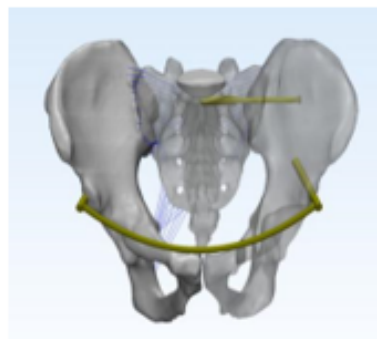

Model7

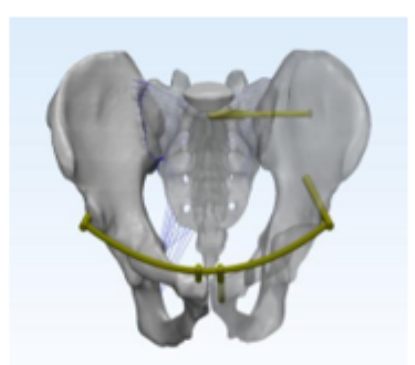

Mode110

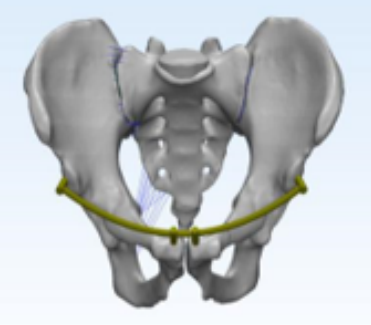

Mode15

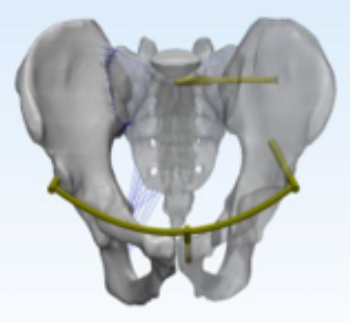

Mode18

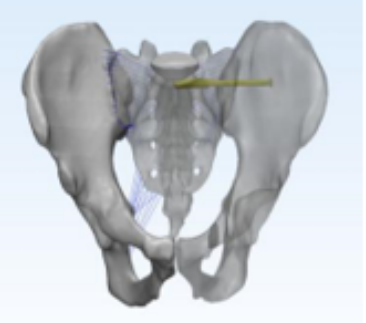

Mode16

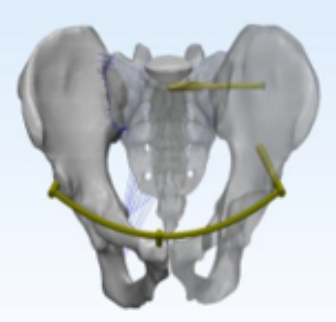

Mode19

\section{Figure 3}

Unfixed pelvis model and 9 kinds of internal fixation pelvis models 